\title{
Two-flavor lattice QCD study of $\Lambda(1405)$
}

\section{Toru T. Takahashi*}

Yukawa Institute for Theoretical Physics, Kyoto University, Sakyo, Kyoto 606-8502, Japan

E-mail: ttoru@yukawa.kyoto-u.ac.jp

\section{Makoto Oka}

Department of Physics, H-27, Tokyo Institute of Technology, Meguro, Tokyo 152-8551 Japan

E-mail: oka@th.phys.titech.ac.jp

\begin{abstract}
Low-lying $\Lambda$ baryons with spin $1 / 2$ are analyzed in two-flavor unquenched lattice QCD. We construct $2 \times 2$ cross correlators from flavor SU(3) "octet" and "singlet" baryon operators, and diagonalize them so that we can extract two low-lying states for each parity. The two-flavor CP-PACS gauge configurations are employed, which are generated in the renormalization-group improved gauge action and the $\mathscr{O}(a)$-improved quark action. Simulation are performed at three different $\beta$ 's, $\beta=1.80,1.95$ and 2.10, whose corresponding lattice spacings are $a=0.2150,0.1555$ and $0.1076 \mathrm{fm}$. For each cutoff, we adopt four different hopping parameters, $\left(\kappa_{\mathrm{val}}, \kappa_{\mathrm{sea}}\right)$. The corresponding pion masses range about from $500 \mathrm{MeV}$ to $1.1 \mathrm{GeV}$. Results indicate that there are two negative-parity $\Lambda$ states nearly degenerate at around $1.6 \mathrm{GeV}$, while no state as low as $\Lambda(1405)$ is observed. By decomposing the flavor components of each state, we find that the lowest (1stexcited) negative-parity state is dominated by flavor-singlet (flavor-octet) component.
\end{abstract}

The XXVII International Symposium on Lattice Field Theory - LAT2009

July 26-31 2009

Peking University, Beijing, China

\footnotetext{
${ }^{*}$ Speaker.
} 


\section{Introduction}

$\Lambda(1405)$ has been attracting much interest from several view points. $\Lambda(1405)$ is the lightest negative-parity baryon, even though it has one valence strange quark in it. Among the $J^{P}=$ $1 / 2^{-}$baryons, $\Lambda(1405)$ is isolated from the others, much lighter than the non-strange counterpart $N(1535)$. It has no spin-orbit partner in the vicinity, as the lowest spin $3 / 2^{-}$state is $\Lambda(1520)$. Furthermore, the structure of $\Lambda(1405)$ remains mysterious. On one hand, $\Lambda(1405)$ is interpreted as a flavor-SU(3)-singlet three-quark state in conventional quark models. On the other hand, $\Lambda(1405)$ could be interpreted as an antikaon-nucleon $\bar{K} N$ molecular bound state (B.E. $\sim 30 \mathrm{MeV}$ ). The binding energy of $\bar{K} N$ implies a strong attractive force between $\bar{K}$ and $N[2,3]$, which may cause a new type of dense hadronic matter, kaonic nuclei or kaonic nuclear matter $[4,5,6]$. We also expect that such $\bar{K} N$ bound states with large binding energies can be regarded as compact 5-quark states. The 5-quark picture of $\Lambda(1405)$ has advantages that all five quarks can be placed in the lowest-lying $L=0$ state to form a negative-parity baryon, and also that it requires no spin-orbit partner of $\Lambda(1405)$.

The property of $\Lambda(1405)$ can therefore be an important clue to new paradigm in hadron physics. We here study properties of $\Lambda(1405)$ using the lattice QCD formulation. Though several lattice QCD studies on $\Lambda(1405)$ have been performed so far $[7,8,9,10]$, most of them are based on quenched QCD and few lattice QCD studies succeeded in reproducing the mass of $\Lambda(1405)$. Moreover, in many cases, individual analyses with "singlet" and "octet" operators were performed, where possible flavor mixings were not taken into account. Little has been discussed on the lattice about the possible mixing of flavor-SU(3)-octet and -singlet components induced by the symmetry breaking. It is then an intriguing issue to clarify the flavor structures in excited-state hadron resonances.

Several possible reasons for the failure of reproducing $\Lambda(1405)$ in lattice QCD were suggested through these studies, such as missing meson-baryon components due to quenching, exotic (non-3quark type) structure of $\Lambda(1405)$, or insufficiency of the lattice volume in the simulations. Resolving such difficulties requires unquenched lattice QCD calculation on a larger lattice volume with varieties of interpolating operators. Then, evaluating contamination by scattering states induced purely by sea quarks would be newly needed. In this report, we aim at clarifying the properties of $\Lambda(1405)$ with two-flavor full lattice QCD, adopting the "octet" and "singlet" baryon operators to construct correlation matrices, which enables us to extract the low-lying spectrum as well as the mixing between octet and singlet components in $\Lambda(1405)$.

\section{Lattice QCD setups}

\section{1 simulation conditions}

We adopt the renormalization-group improved gauge action and the $\mathscr{O}(a)$-improved quark action. We adopt three different $\beta$ 's, $\beta=1.80,1.95$ and 2.10, and corresponding lattice spacings are $a=0.2150,0.1555$ and $0.1076 \mathrm{fm} \mathrm{[11].} \mathrm{We} \mathrm{employ} \mathrm{four} \mathrm{different} \mathrm{hopping} \mathrm{parameters}\left(\kappa_{\mathrm{val}}, \kappa_{\text {sea }}\right)$ for each cutoff. Corresponding pion masses range approximately from $500 \mathrm{MeV}$ to $1.1 \mathrm{GeV}$ at each $\beta$. The details are found in Ref. [1] 


\section{2 baryonic operators}

In order to extract the low-lying states in $S=-1$ and isosinglet channel, we construct $2 \times 2$ cross correlators from the "singlet" and "octet" operators,

$$
\begin{aligned}
& \eta_{\mathbf{1}}(x) \equiv \frac{1}{\sqrt{3}} \varepsilon^{a b c}\left(u^{a}(x)\left[d^{T b}(x) C \gamma_{5} s^{c}(x)\right]+d^{a}(x)\left[s^{T b}(x) C \gamma_{5} u^{c}(x)\right]+s^{a}(x)\left[u^{T b}(x) C \gamma_{5} d^{c}(x)\right]\right) \\
& \eta_{\mathbf{8}}(x) \equiv \frac{1}{\sqrt{6}} \varepsilon^{a b c}\left(u^{a}(x)\left[d^{T b}(x) C \gamma_{5} s^{c}(x)\right]+d^{a}(x)\left[s^{T b}(x) C \gamma_{5} u^{c}(x)\right]-2 s^{a}(x)\left[u^{T b}(x) C \gamma_{5} d^{c}(x)\right]\right)
\end{aligned}
$$

It is easy to check that $\eta_{\mathbf{1}}(x)\left(\eta_{\mathbf{8}}(x)\right)$ belongs to the singlet (octet) irreducible representation of the flavor SU(3). We adopt point-type operators for the sink, $\eta(x)$, and extended operators, which are smeared in a gauge-invariant manner, for the source, $\bar{\eta}(y=0)$. Smearing parameters are chosen so that root-mean-square radius is approximately $0.5 \mathrm{fm}$.

\section{Lattice QCD Results}

\section{1 hadronic masses}

Fig. 1 shows the eigen-energies in the positive- and negative-parity channels, plotted as functions of the squared pion mass $m_{\pi}^{2}$. Filled circles denote the energies of the ground states, and open squares those of 1st-excited states. The solid curves represent quadratic fits as a function of squared pion mass $m_{\pi}^{2}$. Two solid lines at the vertical axes indicate the masses of $\Lambda(1115)$ and $\Lambda(1600)$ in the left panel (positive-parity states), and $\Lambda(1405), \Lambda(1670)$ and $\Lambda(1800)$ in the right panel (negative-parity states).

The obtained masses of the positive-parity ground state agree very well with the mass of the ground-state $\Lambda(1115)$. On the other hand, the 1st-excited state in this channel lies much higher than $\Lambda(1600)$, which is the 1 st excited state experimentally observed so far. The same tendency was reported in Ref. [9], and the situation is similar to the case of the Roper resonance, which is the non-strange SU(3) partner of $\Lambda(1600)$ [12].

In the negative-parity channel (The right panel in Fig.1), the ground- and the 1st-excited states always have close energies at all the $\kappa$ 's. The eigen-energies have similar quark-mass dependences, and the mass splittings are almost quark mass independent. The chirally extrapolated values both lie around the mass of $\Lambda(1670)$ rather than $\Lambda(1405)$. Similarly to the previous studies, the mass of $\Lambda(1405)$ is not reproduced in our calculation. While in quenched simulations in Refs. [7, 8] such failure was regarded as an evidence of possible meson-baryon molecule components in $\Lambda(1405)$, our present unquenched simulation contains effects of dynamical quarks and thus should incorporate meson-baryon molecular states.

We here discuss possible contaminations from scattering states. Since our calculations contain dynamical $u$ and d quarks, scattering states could come into spectra. ( $N \bar{K}$ and $\Sigma \pi$ thresholds are drawn in Fig. 1 as dashed lines.) The energy of the 1st-excited state at the lightest $\mathbf{u}$ - and d-quark masses could be contaminated by such scattering states. There are two meson-baryon channels relevant in the present calculation, $\pi \Sigma$ and $\bar{K} N$. In terms of the valence quark contents, we have 

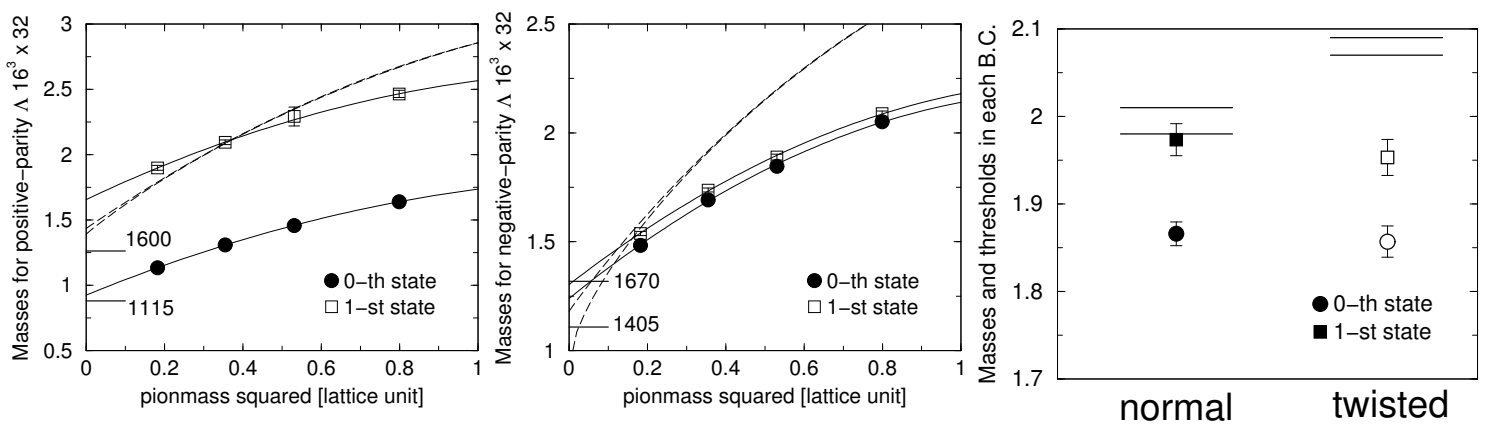

Figure 1: Masses of the lowest two $\Lambda$ states plotted as functions of the squared pion mass. The filled circles (open squares) denote the masses of lowest (1st-excited) state. Two solid curves represent quadratic functions used in the chiral extrapolation. (left panel): Two dashed lines indicate the $\pi \Sigma$ and the $\bar{K} N$ thresholds in the presence of the relative momentum $p=\frac{2 \pi}{L}$. Two solid lines on the vertical axes show the experimentally observed masses of $\Lambda(1115)$ and $\Lambda(1600)$. (middle panel): Two dashed lines indicate the $\pi \Sigma$ and the $\bar{K} N$ thresholds with the relative momentum $p=0$. Two solid lines on the vertical axes show the experimentally observed masses of $\Lambda(1405)$ and $\Lambda(1670)$. (right panel): Lowest two eigen-energies in the $\left(J^{P}, S\right)=\left(1 / 2^{-},-1\right)$ channel under the normal (periodic) and the twisted boundary conditions. The circles (squares) are for the ground (1st-excited) states. The lower and upper solid lines respectively represent the threshold energies of $\Sigma \pi$ and $N \bar{K}$ states evaluated with normal/twisted boundary condition.

5 different thresholds ( 2 for $\bar{K} N, 3$ for $\pi \Sigma$ ). In this report, in order to distinguish resonance states from all these scattering states, we impose the following boundary condition on the quark fields,

$$
\psi(x+L)=e^{\frac{2}{3} \pi i} \psi(x)
$$

Under such boundary condition for quark fields, a hadronic state $\phi_{3 k+n}(x)$ which contains $3 k+n$ valence quarks obeys

$$
\phi_{3 k+n}(x+L)=e^{\frac{2}{3} n \pi i} \phi_{3 k+n}(x),
$$

and can have spatial momenta, $p_{\text {lat }}=\frac{2 \pi}{L} m+\frac{2 n \pi}{3 L} \quad(m \in \mathrm{Z})$.

As a result, only states which consist of $3 \mathrm{k}$ valence quarks can be zero-momentum states. Since other quark combinations inevitably have non-vanishing spatial momenta, their energies are raised up. As long as we employ three-quark operators for baryon creation/annihilation, hadronic states appearing in scattering states should contain one or two valence quark(s), since sea-quark pairs themselves cannot carry flavors.

We plot in Fig. 1 the eigen-energies under the periodic and the twisted boundary conditions. The open and filled circles (squares) are for the ground (1st-excited) states. The lower and upper solid lines respectively represent the threshold energies of $\Sigma \pi$ and $N \bar{K}$ states with normal/twisted boundary condition. The threshold energies are raised up in the case of twisted boundary condition, whereas the lowest two eigen-energies remain unchanged. Thus we conclude that the observed states are insensitive to boundary conditions, and contaminations from meson-baryon scattering states are small, which would be the same reason as the absence of string breaking in heavy-quark potentials from Wilson loops. 


\subsection{Flavor structures}

The chiral unitary approach [13] has suggested that $\Lambda(1405)$ is not a single pole but a superposition of two independent resonance poles. The structure of $\Lambda(1405)$ is now attracting much interest, and desired to be clarified in a model independent manner. We investigate the flavor structures of the ground and the 1st-excited states obtained from the cross correlators of two operators.

In order to clarify the flavor structures of the low-lying states, we evaluate the overlaps of the obtained states with the source and sink operators. We evaluate $g_{0}$ and $g_{1}$ defined as

$$
\begin{aligned}
& g_{0}^{-} \equiv\left\langle 0 \text { th }\left|\eta_{\mathbf{8}}\right| \mathrm{vac}\right\rangle /\left\langle 0 \text { th }\left|\eta_{\mathbf{1}}\right| \mathrm{vac}\right\rangle \\
& g_{1}^{-} \equiv\left\langle 1 \text { th }\left|\eta_{\mathbf{1}}\right| \mathrm{vac}\right\rangle /\left\langle 1 \text { th }\left|\eta_{\mathbf{8}}\right| \mathrm{vac}\right\rangle
\end{aligned}
$$

Both $g_{0}^{-}$and $g_{1}^{-}$vanish when the $\mathrm{SU}(3)_{F}$ symmetry is exact, showing that the ground (1st excited) state is purely flavor singlet (octet) in the limit. For the positive-parity states, we similarly define

$$
\begin{aligned}
& g_{0}^{+} \equiv\left\langle 0 \text { th }\left|\eta_{\mathbf{1}}\right| \mathrm{vac}\right\rangle /\left\langle 0 \mathrm{th}\left|\eta_{\mathbf{8}}\right| \mathrm{vac}\right\rangle \\
& g_{1}^{+} \equiv\left\langle 1 \text { th }\left|\eta_{\mathbf{8}}\right| \mathrm{vac}\right\rangle /\left\langle 1 \mathrm{th}\left|\eta_{\mathbf{1}}\right| \mathrm{vac}\right\rangle
\end{aligned}
$$

In this case, we exchange the denominator and the numerator since the ground state is flavor octet and the 1st excited state is flavor singlet in the $\mathrm{SU}(3)_{F}$ limit.
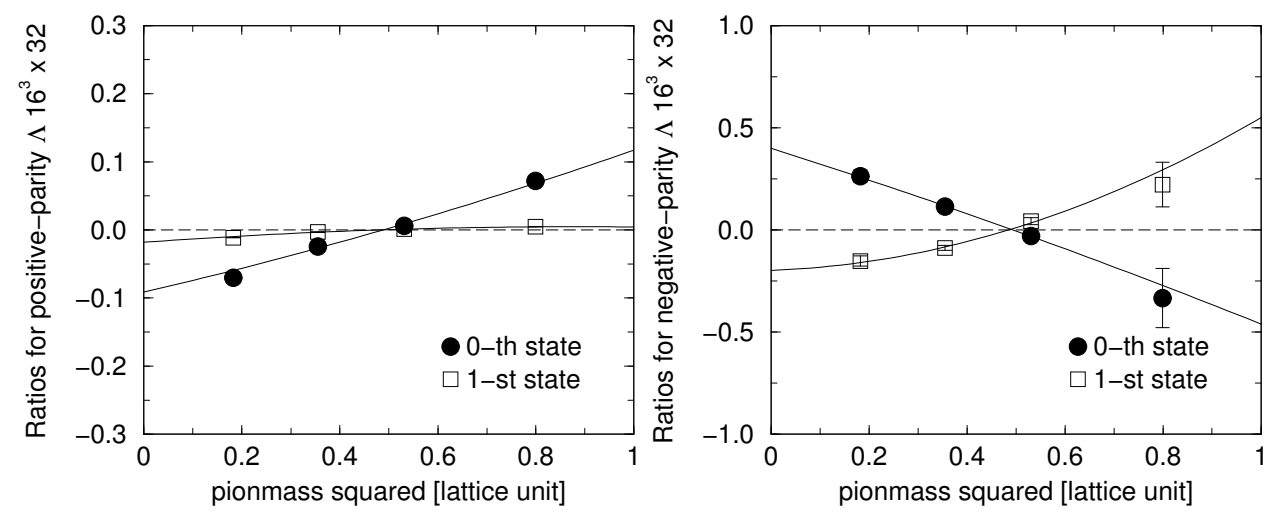

Figure 2: $g_{i}^{ \pm}$are plotted as a function of $m_{\pi}^{2}$. Solid lines denote quadratic functions used in chiral extrapolation. (left panel): The ratio $g_{i}^{+}$in the positive-parity channel. (right panel): The ratio $g_{i}^{-}$in the negative-parity channel.

We show $g_{0}^{ \pm}$and $g_{1}^{ \pm}$as a function of pion-mass squared for each lattice cutoff in Fig. 2. As is expected from the symmetry, such mixing coefficients cross zero at the flavor symmetric limit $\left(\kappa_{u}=\kappa_{d}=\kappa_{s}\right.$ ). The data show smooth quark-mass dependence toward the chiral limit in all the lattice-cut-off cases and the dependences are almost lattice-cut-off independent. We find that the magnitude of operator mixing gets larger for larger flavor-symmetry breaking. It is interesting that the 1st-excited state in positive-parity channel is dominated by the flavor-singlet component showing almost no contaminations of octet components.

On the other hand, the mixings of the singlet and octet components are generally weaker in the positive parity $\Lambda$ 's. It is natural because the splitting of the two states are large in the $\mathrm{SU}(3)_{F}$. 
Nevertheless, it is interesting to see that the ground state, corresponding to $\Lambda(1115)$, contains significant (about 10\%) flavor singlet component in the chiral limit, which was not expected from the simple quark model picture.

\section{Discussions}

Our results for negative-parity $\Lambda$ states indicate that there are two $1 / 2^{-}$states nearly degenerate at around $1.6-1.7 \mathrm{GeV}$, while no state as low as $\Lambda(1405)$ is observed. We have revealed the flavor structures of these states from the lattice data for the first time. It is found that the lowest negativeparity $\Lambda$ state is dominated by flavor-singlet component. The second state, which is less than 100 $\mathrm{MeV}$ above the ground state, is mainly flavor octet. We thus find that the first two negative-parity $\Lambda$ 's have different flavor structures.

They, however, come significantly heavier than the experimentally observed lowest-mass state, $\Lambda(1405)$. Considering that our simulation contains two-flavor dynamical quarks, the failure of obtaining a light $\Lambda$ state could be attributed either to (1) strange-quark quenching, (2) insufficient lattice volume or (3) lack of chiral symmetry. In fact, (1) seems to make the masses of octet baryons in positive parity channel slightly $(\leq 10 \%)$ overestimated in the present setups [11]. On the other hand, the deficiencies,(2) and (3), may cause the lowest state not properly reproduced, supposing that the main component of $\Lambda(1405)$ is a meson-baryon molecular state. In order to check whether this conjecture is correct, simulations with light dynamical quarks $\left(m_{\pi} \ll 500 \mathrm{MeV}\right)$ and larger volume $(L \gg 2.5 \mathrm{fm})$ will be required.

One may ask whether one or both of the obtained states in the present calculation correspond to the physical $\Lambda(1405)$. One possible scenario is that they correspond to the physical $\Lambda(1405)$ and $\Lambda(1670)$, whose masses become larger due to the deficiencies discussed above. While this scenario is simple and probable, we here consider two other possibilities. First, suppose that these states correspond to the physical $\Lambda(1405)$, then the results may support its double pole structure proposed by the chiral unitary approach [13]. In our results, the lowest two states are almost degenerate at all the $\beta$ 's (lattice spacing) and $\kappa$ 's (quark masses). Namely, the obtained two states are the signature of the double-pole resonance, but the mass has not yet been reproduced because of the deficiency stated above.

The other possibility is that the obtained states do not correspond to the physical $\Lambda(1405)$, but describe excited $\Lambda$ states. In fact, the 2 nd and 3rd negative-parity states lie at $1670 \mathrm{MeV}$ and $1800 \mathrm{MeV}$, both of which are three-star states in the Particle Data Group classification. The present lattice data are consistent with these excited states. In the non-relativistic quark model approach, each of these states is classified as a flavor octet P-wave baryon. We, however, have shown that the lower state is dominated by a flavor-singlet component. So, the results here predict one flavorsinglet state and one flavor-octet state in the vicinity.

The flavor structures of the $\Lambda$ states can be well clarified using the variational method. The octet and the singlet components are mixed when the flavor-SU(3) symmetry is broken: The ground (1st-excited) state is dominated by singlet (octet) component, and the contamination by another representation is at most 20\% (5\% when squared) in our present analysis. From these findings, we expect that the flavor-SU(3) symmetry is not largely broken. A similar conclusion was also derived for the study of the meson-baryon coupling constants in lattice QCD [16]. Because the SU(3) 
breaking effect seems not so large, the analyses without $\mathrm{SU}(3)$ mixings adopted so far $[7,8,9,10]$ make sense to some extent. The mixings, however, get larger towards the chiral limit, variational analyses could be essentially needed when we adopt much lighter quarks.

\section{Acknowledgments}

All the numerical calculations were performed on NEC SX-8R at CMC, Osaka university and BlueGene/L at KEK. The unquenched gauge configurations employed in our analysis were all generated by CP-PACS collaboration [11]. This work was supported in part by the 21st Century COE 'Center for Diversity and University in Physics", Kyoto University and Yukawa International Program for Quark-Hadron Sciences (YIPQS), by the Japanese Society for the Promotion of Science under contract number P-06327 and by KAKENHI (17070002, 19540275, 20028006 and 21740181).

\section{References}

[1] T. T. Takahashi and M. Oka, arXiv:0910.0686 [hep-lat].

[2] J. J. Sakurai, Annals Phys. 11, 1 (1960).

[3] R. H. Dalitz, T. C. Wong and G. Rajasekaran, Phys. Rev. 153, 1617 (1967).

[4] Y. Akaishi and T. Yamazaki, Phys. Rev. C 65, 044005 (2002).

[5] T. Yamazaki and Y. Akaishi, Phys. Lett. B 535 (2002) 70.

[6] Y. Akaishi, A. Dote and T. Yamazaki, Phys. Lett. B 613, 140 (2005) [arXiv:nucl-th/0501040].

[7] W. Melnitchouk et al., Phys. Rev. D 67, 114506 (2003) [arXiv:hep-lat/0202022].

[8] Y. Nemoto, N. Nakajima, H. Matsufuru and H. Suganuma, Phys. Rev. D 68, 094505 (2003) [arXiv:hep-lat/0302013].

[9] T. Burch, C. Gattringer, L. Y. Glozman, C. Hagen, D. Hierl, C. B. Lang and A. Schafer, Phys. Rev. D 74, 014504 (2006) [arXiv:hep-lat/0604019].

[10] N. Ishii, T. Doi, M. Oka and H. Suganuma, Prog. Theor. Phys. Suppl. 168, 598 (2007) [arXiv:0707.0079 [hep-lat]].

[11] A. Ali Khan et al. [CP-PACS Collaboration], Phys. Rev. D 65, 054505 (2002) [Erratum-ibid. D 67, 059901 (2003)]

[12] S. Sasaki, T. Blum and S. Ohta, Phys. Rev. D 65, 074503 (2002) [arXiv:hep-lat/0102010].

[13] D. Jido, J. A. Oller, E. Oset, A. Ramos and U. G. Meissner, Nucl. Phys. A 725 (2003) 181 [arXiv:nucl-th/0303062].

[14] U. M. Heller, K. M. Bitar, R. G. Edwards and A. D. Kennedy, Phys. Lett. B 335, 71 (1994) [arXiv:hep-lat/9401025].

[15] B. Bolder et al., Phys. Rev. D 63 (2001) 074504 [arXiv:hep-lat/0005018].

[16] G. Erkol, M. Oka and T. T. Takahashi, Phys. Rev. D 79, 074509 (2009) [arXiv:0805.3068 [hep-lat]]. 